IOS Press

\title{
Erratum
}

\section{Stimulation of functional recovery via the mechanisms of neurorepair by S-nitrosoglutathione and motor exercise in a rat model of transient cerebral ischemia and reperfusion}

Harutoshi Sakakima, Mushfiquddin Khan*, Tajinder S. Dhammu, Anandakumar Shunmugavel, Yoshihiro Yoshida, Inderjit Singh and Avtar K. Singh

[Restorative Neurology and Neuroscience 30(5), 2012, 383-396, DOI 10.3233/RNN-2012-110209] http:// iospress.metapress.com/content/h5hl216523587370/

Page 393: Due to an error in the assembling of photomicrographs (Fig. 8) showing the expression of Akt for GSNO, EX + LY and GSNO + LY, an incorrect version of Fig. 8 was originally published in the above article. The corrected Fig. 8 appears below. The change does not affect the results, the interpretation and the conclusion of the reported study.

*Corresponding author: Mushfiquddin Khan, E-mail: khanm@ musc.edu. 


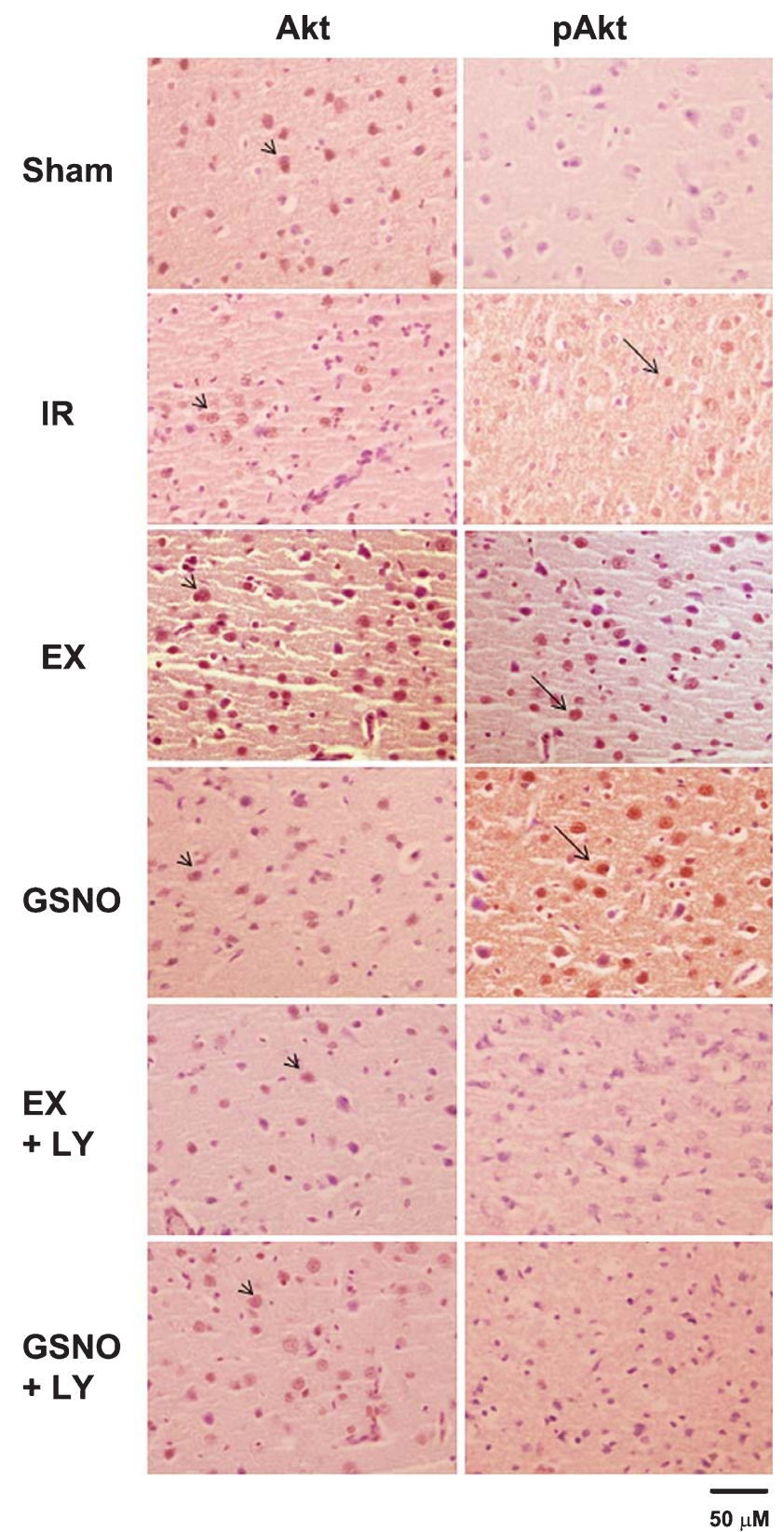

Fig. 8. Photomicrographs of DAB staining of immunoreactivity of Akt and pAkt at day 7 after IR. 